\title{
Pathological mechanosensing
}

\author{
A deeper understanding of the myriad ways that the mechanics of cellular and tissue microenvironments trigger or \\ exacerbate disease will open up pathways for new interventions.
}

A therosclerosis comes with the hardening of the arteries. Fibrosis in the liver and lung is exacerbated by the stiffness of the extracellular matrix. Many types of tumours are stiffer than the surrounding healthy tissue (cancer cells, however, are typically softer). And plenty of disease-triggered tissue abnormalities can be diagnosed by palpation (manual or via surgical probes).

However, cellular and tissue mechanopathology have been explored with much less intensity than the molecular and cellular biology and the biochemistry of disease, partly because of rapid developments in DNA sequencing and cloning, in microarray analysis and in mass spectrometry. However, the information encoded in DNA and the protein-expression profiles of cells do not fully determine cellular phenotypes and tissue function. Cells and tissues are continuously exposed to forces in their environment - such as shear stresses caused by blood flow or respiration, and compression and tension forces from body movement - and respond to them both mechanically and biochemically. In fact, cells sense the mechanics of their environment (the extracellular matrix, neighbouring cells, surfaces, and cellular and tissue interfaces) via a range of mechanosensitive proteins or protein complexes (such as stretch-responsive ion channels, adhesion complexes, cell-cell junctions and the cytoskeleton), which can activate a wide range of intracellular signalling pathways that ultimately (via the nuclear translocation of transcription factors and even the post-translational modification of regulatory proteins) result in the altered expression or repression of 'mechanosensitive' genes.

Hence, the mechanics of the cellular microenvironment contribute to cellular phenotypes, and in turn the microenvironment is shaped by the cells themselves. Yet, for every particular disease condition, how much, and under which conditions, do transduced mechanical cues contribute to the disease? This issue compiles seven studies that further the understanding of cellular mechanosensing and that point to possible interventions for the amelioration of disease.

The mechanical properties of the cellular microenvironment modulate the function

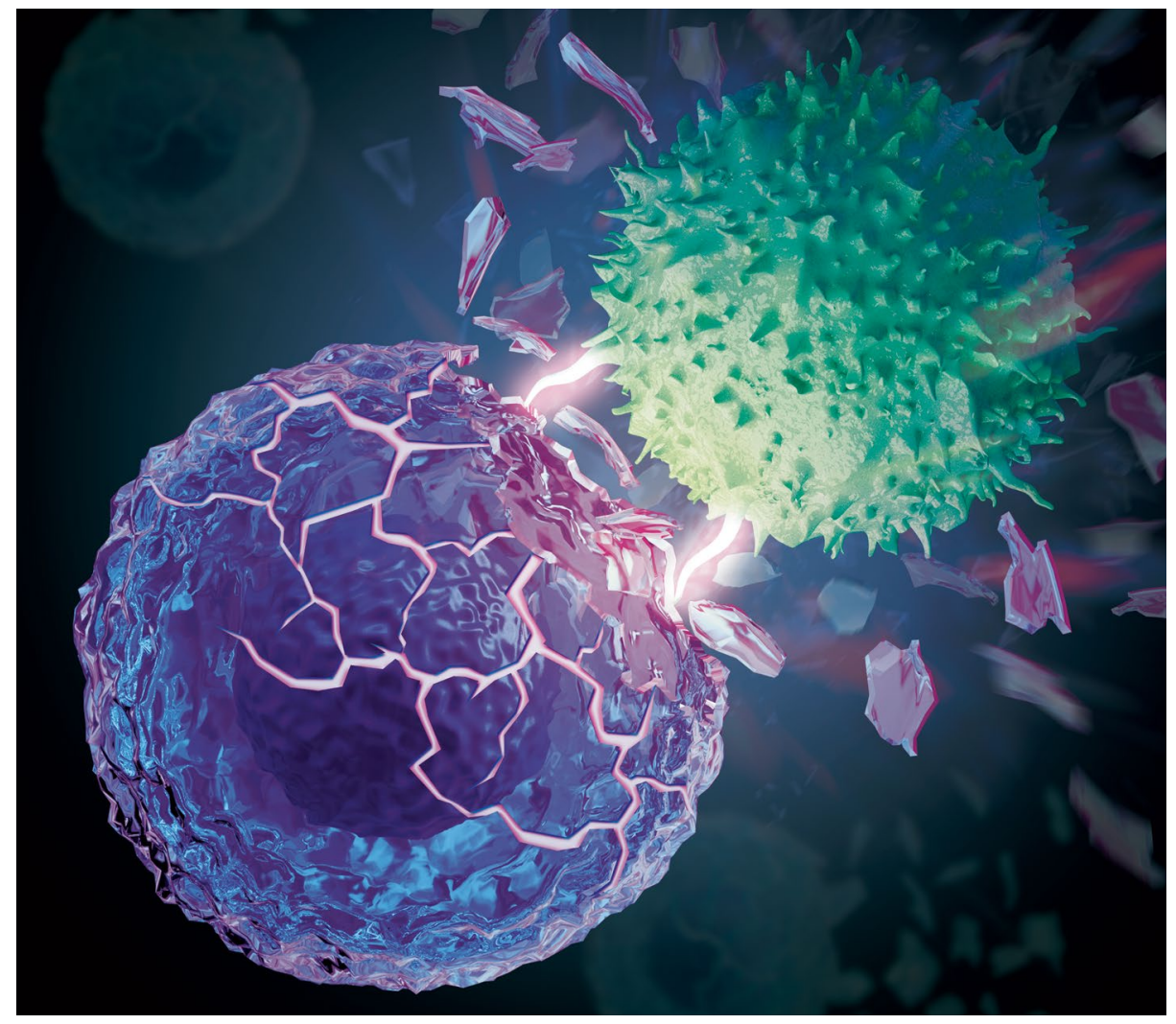

Credit: Image courtesy of My Scimage, and Kewen Lei and Li Tang at EPFL.

of T cells. Also, because T-cell receptors are mechanosensitive, forces taking place at the immunological synapse formed between $\mathrm{T}$ cells and antigen-presenting cells can regulate the potency of cytotoxic T cells. $\mathrm{Li}$ Tang and colleagues found that the softness of cancer cells impairs the mechanics at the synapse, conferring the cells with resistance to T-cell-mediated cytotoxicity. They also show that this 'mechanical immune checkpoint' (pictured) could be therapeutically targeted by stiffening the membrane of cancer cells through cholesterol depletion (via the solubilizer methyl- $\beta$-cyclodextrin), as they show in mouse models of adoptive T-cell therapy.

The compression of blood vessels and lymphatics by tumours can cause clinical symptoms. Cancer cells can take advantage of such solid stresses to metastasize to lymph nodes and proliferate in them, as Dennis Jones, Timothy Padera and co-authors report. By examining metastatic lymph nodes from patients with a variety of cancer types, as well as mice with spontaneously developing breast-cancer lymph-node metastases, they found that growing metastatic lesions in lymph nodes generate compressive solid stresses that impair the entry of $\mathrm{T}$ cells, making the metastases 'immunologically cold' and hence resistant to immunotherapies such as immune checkpoint inhibitors. They also show that reducing solid stress in the metastatic lesions by inhibiting the synthesis of collagen (via the angiotensin-receptor-blocker losartan) enables the entry of T cells. Limiting metastatic growth in lymph nodes may thus reduce the number of cancer cells travelling from the lymph nodes to distant organs.

In cartilage, alterations in the mechanical properties of the extracellular matrix are implicated in osteoarthritis. Nidhi Bhutani, Ovijit Chaudhuri and colleagues investigated how the viscoelasticity of the matrix affects osteoarthritic chondrocytes. They 
found that a particular molecular pathway (involving an ion-channel protein and glycogen synthase kinase) is dysfunctional in osteoarthritic chondrocytes, preventing them from sensing changes in matrix viscoelasticity and thus from regulating their volume, which primes these cells for enhanced responses to inflammatory cues. They also show that treating aged healthy chondrocytes with an inhibitor of the ion channel involved in the dysfunctional pathway protected the chondrocytes from acquiring an osteoarthritic phenotype.

As with cartilage, tendons are also load-bearing tissue, yet how they sense physical forces and adapt to them was unknown. Jess Snedeker and colleagues reveal that the mechanosensitive ion channel PIEZO1 senses shear stresses in tenocytes, leading to increases in the strength and stiffness of tendon tissue via the upregulation of collagen crosslinking. They show that the stiffness of tendon is reduced when the activity of PIEZO1 is inhibited via loss-of-function experiments (and augmented when the mechanosignalling activity of PIEZO1 is elevated) and that, intriguingly, individuals carrying a PIEZO1 gain-of-function mutation (common in individuals of African descent) performed better at jumping (demonstrating a more than $10 \%$ greater normalized jumping height). As noted by Spencer Lake and co-authors in an accompanying News and Views, PIEZO1-induced crosslinking is likely to be one factor among many that influence how tendons adapt in response to mechanical loading; yet, an improved understanding of how tendon properties are regulated through PIEZO1 could help to inform physical therapy for improving the healing of injured tendons.

Stiffer tissue can also be triggered by implanted biomaterials. Collagen deposition triggered by the responses of tissues to 'foreign bodies' leads to their fibrotic encapsulation. Boris Hinz and co-authors found that the stiffness of the surface of silicone breast implants contributes to the activation of myofibroblasts and to the development of fibrosis in a cascade of events involving larger intracellular stresses, the recruitment of integrins and the activation of signalling by latent pro-fibrotic transforming growth factor $\beta 1$. They also show that the activation of the latent growth factor can be abrogated by a small-molecule integrin inhibitor or by reducing the stiffness of the implant surface via a soft coating layer. In a News and Views highlighting the work, Stuart Bauer and Joshua Doloff posit that the soft-coating strategy may also mitigate the fibrotic encapsulation of implanted sensors and pacemakers and that soft coatings could be adapted to protect drug-eluting devices and cell-encapsulating devices.

The pathogenic deposition of extracellular matrix by myofibroblasts that are persistently activated also underlies fibrotic disease more generally. Kristi Anseth and colleagues found that the persistent activation of myofibroblasts in fibrotic tissue is manifested in the cell's nucleus in the form of chromatin that is more 'condensed' (as compared to that of transiently activated myofibroblasts), and that this is caused by the direct transmission of forces from the extracellular matrix to the nucleus via the cytoskeleton; in fact, the inhibition of actin polymerization prevented the persistent activation of the myofibroblasts.

Because of its abundant interconnections with the cell's cytoskeleton, the cell's nucleus plays a central role in mechanosensing. Corey Neu and co-authors show that, in addition to integrating environmental mechanical cues, the nucleus in cardiomyocytes stabilizes the cell's fate through the reorganization of chromatin marked by trimethylation of histone 3 lysine 9 towards the nuclear border, and that altered nuclear deformation through the exposure to stiffer substrates or stiffer diseased tissue can result in the loss of distinct chromatin organization, which may be of diagnostic value.

Unfortunately, rarely can a single pathway be silenced or boosted neatly and robustly, and even then, phenotypic plasticity may override any effects of the treatment. It may also be difficult to find biomarkers for quantifying the efficacy of the treatment, as has been the case with antiangiogenic integrin-targeted therapeutics. Hence, in addition to the identification and understanding of cellular mechanosensory pathways, it is crucial to investigate how they engage in 'crosstalk' with biochemical signalling implicated in disease - from rare genetic disorders such as laminopathies to inflammation in the 'wound' created by an implant. Such knowledge should facilitate the design of effective 'mechanotherapeutics' and of fibrotically stealthy implantable biomaterials.

Published online: 13 December 2021 https://doi.org/10.1038/s41551-021-00835-5 